\title{
A Distributed Virtual Time System on Embedded Linux for Evaluating Cyber-Physical Systems
}

\author{
Christopher Hannon, Jiaqi Yan, Yuan-An Liu \\ Illinois Institute of Technology \\ Chicago, Illinois \\ \{channon,jyan31,yliu301\}@hawk.iit.edu
}

\author{
Dong Jin \\ Illinois Institute of Technology \\ Chicago, Illinois \\ dong.jin@iit.edu
}

\begin{abstract}
Cyber-physical systems have a cyber presence, collecting and transmitting data, while also collecting information and modifying the physical surrounding world. In order to evaluate the cyber-security of cyber-physical systems, simulation and modeling is a tool often used. In this work, we develop a distributed virtual time system that enables the synchronization of virtual clocks between physical machines enabling a high fidelity simulation based testing platform. The platform combines physical computing and networking hardware for the cyber presence, while allowing for offline simulation and computation of the physical world. By incorporating virtual clocks into distributed embedded Linux devices, the testbed creates the opportunity to interrupt real and emulated cyber-physical applications to inject offline simulated data values. The ability to run real applications and being able to inject simulated data temporally transparent to the running process allows for high fidelity experimentation. Distributed virtual time enables processes and their clocks to be paused, resumed, and dilated across embedded Linux devices through the use of hardware interrupts and a common kernel module. By interconnecting the embedded devices' general purpose IO pins, they can coordinate and synchronize through a distributed virtual time kernel module with low overhead, under 50 microseconds for 8 processes across 4 embedded Linux devices. We demonstrate the usability of our testbed in a power grid control application.
\end{abstract}

\section{KEYWORDS}

Embedded Linux, Synchronization, Cyber-Physical Systems

\section{ACM Reference Format:}

Christopher Hannon, Jiaqi Yan, Yuan-An Liu and Dong Jin. 2019. A Distributed Virtual Time System on Embedded Linux for Evaluating CyberPhysical Systems. In SIGSIM Principles of Advanced Discrete Simulation (SIGSIM-PADS '19), June 3-5, 2019, Chicago, IL, USA. ACM, New York, NY, USA, 12 pages. https://doi.org/10.1145/3316480.3322895

\footnotetext{
Permission to make digital or hard copies of all or part of this work for personal or classroom use is granted without fee provided that copies are not made or distributed for profit or commercial advantage and that copies bear this notice and the full citation on the first page. Copyrights for components of this work owned by others than ACM must be honored. Abstracting with credit is permitted. To copy otherwise, or republish, to post on servers or to redistribute to lists, requires prior specific permission and/or a fee. Request permissions from permissions@acm.org.

SIGSIM-PADS '19, June 3-5, 2019, Chicago, IL, USA

(C) 2019 Association for Computing Machinery.

ACM ISBN 978-1-4503-6723-3/19/06 .. \$15.00

https://doi.org/10.1145/3316480.3322895
}

\section{INTRODUCTION}

Embedded computers today are transformed from self-contained systems to various cyber-physical systems (CPS) by sensing, monitoring, controlling our physical world. A sound evaluation of those systems as well as the applications running on top of them is essential but highly challenging. As embedded computers monitor and control mission critical physical processes in real-time (e.g., an electrical power system), performing an evaluation on the actual system is often disabled to avoid interference with normal system operations. Virtual testbeds are tools designed to address this challenge. A capable testbed combines both physical and virtual components, including but not limited to real embedded devices, virtual machines, emulated communication networks, simulation models of physical processes, analytical models of background traffic, etc.

A key challenge in simulating CPS is to seamlessly combine the physical and virtual worlds to conduct high-fidelity experiments, as real components execute applications with the real world wall clock and virtual components advance model states with a virtual clock. One solution is to provide a notion of virtual time to the physical processes so that their executions can be explicitly scheduled with simulation models and advance together in virtual time. Virtual time is a concept that was designed to enable multiple virtual machines to be multiplexed on a single physical hardware. We can use virtual time to schedule sequentially executed processes so that from their perspective they are being run in parallel. In simulation and modeling, virtual time is a technique used to synchronize emulated processes to make them execute in a reproducible way and behave more like traditional simulation models [16-18], which also enables simulation models to be integrated with emulation. Furthermore, virtual time can also be used to slow down a running process and thus increase the perception of resources [29, 30]. As a result, emulated processes can be executed on hardware that has fewer resources than required by the processes. For example, in communication network emulation, bandwidth on a virtual link can exceed the physical bandwidth of the hardware by slowing down the processes' perception of time by some time dilation factor (TDF) [9].

A number of virtual time systems have been developed for different types of virtual machines running on a single physical machine (e.g., Xen [9], Linux container [18], and OpenVZ [13]). Taking a set of processes and programs and using virtual time to schedule their execution, one can enable fine-grained control over the execution and interaction of processes. These sets of processes can be merged with traditional simulation systems (e.g., communication networks $[19,30]$ and power grids [10]) using virtual time to integrate high fidelity executing processes with simulation models. 


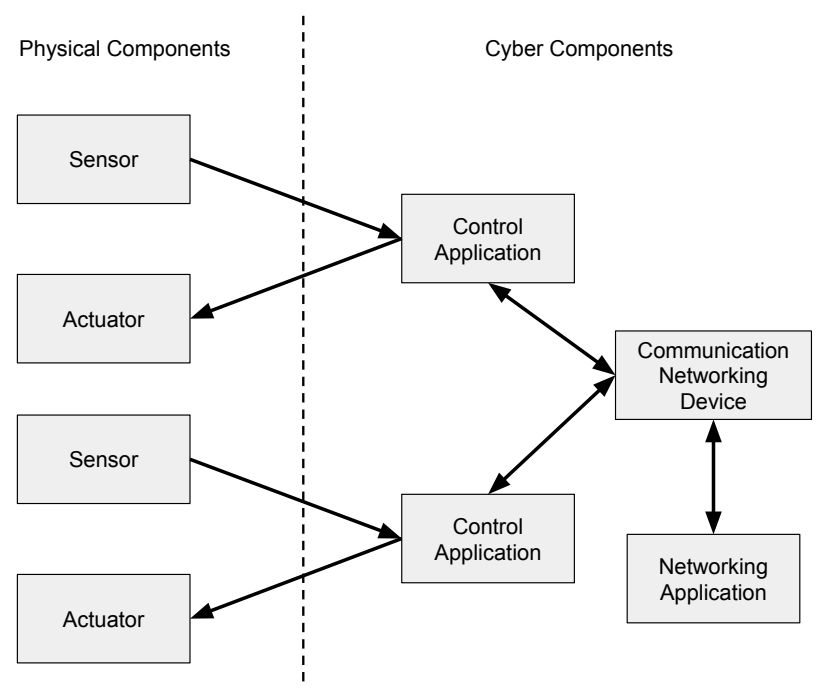

Figure 1: Overview of a general cyber-physical system. Distributed virtual time enables the integration of the simulation of the physical state with the emulation using real hardware and processes of the control applications and communication networks.

In this work, we further enhance the capacity of a virtual testbed by developing the first distributed virtual time system on embedded Linux. The system enables efficient synchronization between the simulation of the physical aspect of CPS and real hardware computing devices running embedded Linux. Our contribution is a distributed system architecture uniquely consisting of a common virtual time Linux kernel module and three communication channels, one for virtual time synchronization using general-purposeinput-and-output (GPIO) hardware interrupts, one for connecting the embedded Linux devices, and one for interfacing with the physical system simulation that performs an offline computation. Rather than integrate emulated processes with simulation models, our work leverages the concept of virtual time to perform the reverse, namely to take simulation systems and integrate them into emulated systems. This distributed virtual time system enables the creation of a CPS testbed that can run real emulated processes while simulating the effects of the physical system.

Considering the electric power grid for example, sensors feed data into control applications, which interact and in turn send control signals back to actuators to modify the state for the cyberphysical system. Figure 1 illustrates a general CPS. The left side represents the physical component of the system while the right side represents the cyber component of the system. The distributed virtual time system enables the establishment of a high-fidelity hardware-in-the-loop testbed, with which we can simulate the state of the physical world including the sensors and actuators, and inject the data into emulated control processes running across a distributed platform.

Our implementation works for various embedded Linux devices with GPIO programmability, such as the Banana Pi M1s, the Banana Pi R1 Routers, and the Raspberry Pi devices. To demonstrate the practicality of distributed virtual time, we measure the system overhead with the modified kernel (e.g., modifying the gettimeofday system call to enable processes to query their virtual clocks raises the overhead from 11.8 to just 17.4 microseconds, while a page fault takes over 100 microseconds) and evaluate the correctness of virtual time across multiple hardware devices concerning clock skewness and network application performance. To demonstrate the usability of the distributed virtual time system, we integrate the distributed virtual time testbed with a power grid simulator and present a case study to evaluate the cyber-security of a voltage stabilization application.

The rest of the paper is organized as follows, Section 2 provides the background, the design of virtual time, and the synchronization challenges. Section 3 shows in detail how to implement a distributed virtual time system. In Section 4, we evaluate the correctness and performance of our virtual time system. We present a use case of our testbed in Section 5. In Section 6 we show related work in combining simulation and emulation as well as other uses of virtual time. Finally, we conclude with future work in Section 7.

\section{VIRTUAL TIME DESIGN}

Virtual time is a centrally coordinated system which interfaces with running processes to provide an altered perception of time. We design a virtual time system running in synchronization across a distributed platform.

\subsection{Background of Virtual Time in Testbeds}

When a process registered with virtual time requests the current time, the virtual time system transparently reports a virtual time. When processes are placed in a paused state, they are not running nor are their virtual clocks advancing. Upon resuming, processes are placed in the running state and their clocks advance with the virtual clock. Virtual time has two abilities, the first is to pause and resume virtual-time-enabled processes and the second is to dilate the running clock to either make a processes perception of virtual time faster or slower than the real wall clock. Our work strongly utilizes the first ability but supports the ability to scale a process' perception as well.

The contribution of our work is the design of a physically distributed but centrally coordinated virtual time system for cyberphysical systems. In order to synchronize the simulated and real parts of the cyber-physical testbed, the pausing and resuming of processes on real/emulated devices is necessary. The synchronization problem is that emulation and real hardware applications are running in wall-clock time while the physical components of the system are simulated. The simulation uses a simulation clock which may advance at a slower rate than the wall clock. For example, a sensor can take a measurement very quickly in real life, but in simulation, the state of the simulation may take much longer to advance. By utilizing a virtual time system between running processes and the operating system's clock, the virtual time system can modify processes' perception of time transparently to the running process. For CPS, virtual time can ensure the accuracy of data acquisition protocols. As an example, sensor data reading frequency may be rate-limited by the communication channel bandwidth. Virtual time 
can ensure that the bandwidth remains consistent with real world behavior.

When a process subscribed to virtual time requests the current time, the operating system returns the process' virtual time, which is equal to the wall clock time less the time since the process was started, less total time paused, scaled by the time dilation factor and then added back to the time that the process started. For example, given start time $T_{s}$, current wall clock time $T_{w c}$, time dilation factor $t d f$, and total cumulative time paused $T_{p}$, the process's virtual time, $T_{V T}$ is given by the formula:

$$
T_{V T}=\frac{T_{w c}-T_{s}-T_{p}}{t d f}+T_{s}
$$

Therefore, when a process requests the current time, the process receives the total time running (while not paused) scaled by the dilation factor. Our design objective is to make this time function transparent to any process so that no code modification is required for a process to use virtual time.

\subsection{Distributed Virtual Time}

However, synchronization challenges emerge when applying virtual time to processes running across distributed hardware. Figure 2 illustrates the desired behavior for a distributed virtual time system with $n$ devices. The $x$ axis shows the time with respect to the wall clock time. The black lines illustrate when a process and its clock is advancing. When a process on any device, i.e., Device 1 requests an offline computation from some external simulation source, all processes across devices using virtual time should be paused. Similarly, when the offline computation completes, all processes should resume uniformly. At time $T 1$, a virtual time enabled process on Device 1 makes a synchronization request as shown by the red solid arrow. This request triggers the virtual time system to pause or freeze all the clocks and processes that are virtual time enabled across all devices. At time T2, the processes and their clocks are paused. Between times $T 2$ and $T 3$, the offline computation request

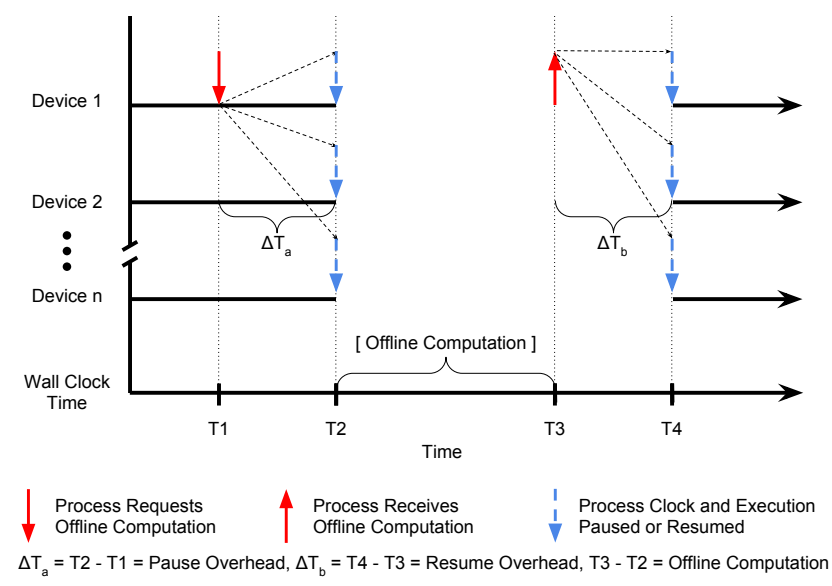

Figure 2: A high level design of distributed virtual time synchronization. When a virtual time process requests an offline computation, processes across all devices are paused and resumed uniformly. is executed. Upon receiving a reply at time $T 3$, a notification is sent to the virtual time system on Device 1 . At time $T 4$, all virtual time processes and their clocks resume. Ideally, $\Delta T_{a}$ and $\Delta T_{b}$, the overheads of the pause and resume routines should be zero. Additionally, the pause and resume operations (T2 and T3) must occur across devices at the same wall clock time to ensure virtual time synchronization. In Figure 2, the total virtual time that has elapsed for the processes on Devices $[1: n]$ is the wall clock time less $T 4-T 2$, which is the total paused time.

In order to synchronize virtual time across multiple hardware devices, a communication channel is needed to connect the virtual time system on each device to distribute the pause and resume signals. However, in order to maintain high accuracy, the communication channel must have minimal latency. Shared channels, such as Ethernet, have too large of an overhead and rely on system scheduling. A dedicated link is ideal to prevent any congestion. Additionally, since the cyber-physical system itself relies on a communication network, this network must be disjoint from the virtual time system's communication channel ensuring accuracy. Finally, a third communication channel is needed to interface with an external compute engine for offline computations including simulating the physical components of the CPS.

Our intuition for developing a distributed virtual time system is to use hardware interrupts to coordinate and synchronize virtual time across devices. Each device runs a common virtual time system, which communicates to each other via hardware interrupts and voltage signals. The design of the virtual time system must ensure that any process on any device is able to pause the virtual time across all devices in real time. The design of this virtual time system must be highly responsive in order to ensure this requirement. Hardware interrupts are very fast and can be set to call a handler function upon changes on an incoming voltage to a physical pin on the hardware device. Because many devices (e.g., sensors) in cyber-physical systems are low power, we utilize embedded Linux devices, which enables us to construct a low-cost hardware and emulation testbed while maintaining high fidelity.

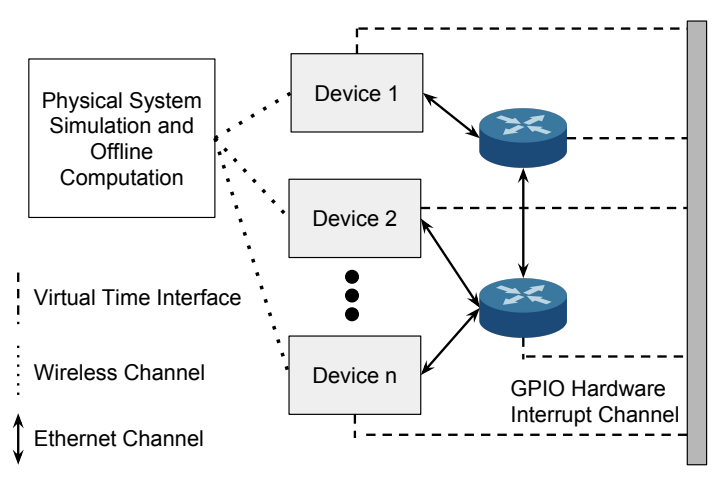

Figure 3: The architecture of the virtual time system enables a virtual time channel, a channel for the testbed, as well as a channel to interface with a source for simulating the physical component of the CPS. All physical devices are required to be virtual time enabled. 
Figure 3 illustrates our design of a virtual time enabled simulation and emulation testbed. Devices can be machines that have a cyber and physical presence or just a cyber presence. The devices are Linux machines and are networked together through embedded Linux routers. All the physical devices run an instance of the distributed virtual time system, and any of them can have a connection to an external simulation and offline computation system.

Furthermore, our design of distributed virtual time must account for multiple hosts making offline requests at the same time or nearly at the same time. In Figure 4, we illustrate the desired behavior. When processes request an offline computation at nearly the same time, it can be possible that two requests are sent before the first one pauses the system. For example, this scenario can occur when sensors collect data at synchronized periodic increments. While virtual time supports parallel requests, the power simulator executes requests sequentially. If this occurs, we must ensure that the virtual time enabled processes are paused correctly. All processes should not be resumed until all pending events are finalized. Therefore, for $n$ processes $p_{i}$ on all the devices in the testbed, we define a binary function $S(p)$ such that $S\left(p_{i}\right)=1$ maps to a request for process $p_{i}$, otherwise $S(p)=0$. The pausing criteria becomes

$$
\sum_{i=0}^{n} S\left(p_{i}\right) \geq 1
$$

while the resuming criteria is

$$
\sum_{i=0}^{n} S\left(p_{i}\right)=0
$$

In Figure 4, at time $T 1$, a request is created on Device 1 . However, at time $T 1^{\prime} \leq T 2$, another request is created on Device $n$ before the virtual time system completes the pause routine. Therefore, instead of resuming at time $T 3$ as in Figure 2, the virtual time system must enforce the system to resume at time $T 3^{\prime}$ when all pending synchronization requests are completed.

\section{IMPLEMENTATION}

\subsection{Distributed Virtual Time Enabled Emulation}

Our emulation testbed supports distributed virtual time. By design, any physical host is allowed to play the role of the virtual time master during experiment run time to initiate synchronization requests (i.e., pause or resume the emulation) to all the running hosts. We illustrate the system using a four-host scenario as shown in Figure 5, where Host 1 sends the synchronization request to the other three hosts.

For example, Host 1 issues the request to pause the distributed emulation testbed; the request is handled by the virtual time Linux kernel module (LKM); the OS kernel eventually converts it to a hardware signal. In the case of pause, the kernel changes the state on one of Host1's general purpose input and output (GPIO) pins to HIGH. This change outputs a voltage signal (the red signal in Figure 5). Since all the hosts including the master Host 1 are connected to the voltage signal bus, they can read the voltage change. Each host's OS kernel has an interrupt registered to a rising/falling voltage signal on two of its hardware GPIO pins (different from the output pin). Depending on the type of the signal, the kernel triggers

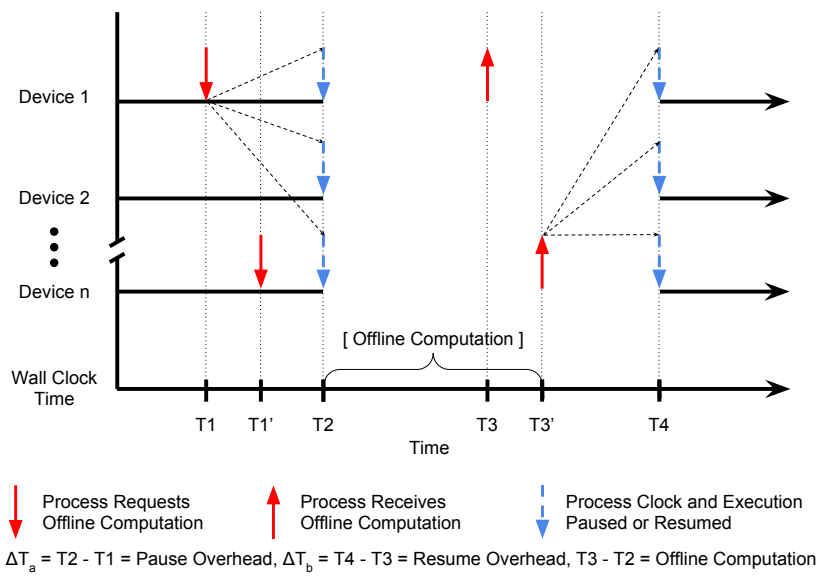

Figure 4: When two processes on different devices create a synchronization event simultaneously, both virtual time systems handle the request. The processes on all devices should pause their clocks when receiving the synchronization event, however, should only resume when all pending offline computation events are completed. This is accomplished by utilizing voltage signals on a common bus.

a software interrupt that the virtual time LKM has registered with an associated software routine. In the case of a rising voltage, the triggered software interrupt instructs the virtual time module to pause all processes subscribed to the virtual time system. Resuming applications in virtual time work similarly except that a falling voltage signal (the blue signal in Figure 5) is created by changing the output pin's state on the virtual time master from HIGH back to LOW. The change in signal then triggers an interrupt on the pins registered to the falling voltage. Due to the oscillations in the electronic signal change, a debounce time of 20 microseconds is required on the rising and falling interrupts. In our future work, we will explore hardware debouncers to reduce this time. The hardware interrupts are configured and triggered using the generic interrupt controller (Corelink GIC-400) [1] which connects peripherals to the A20 Allwinner CPU [2]. The rising and falling interrupts are registered to separate pins due to this configuration.

Apart from initially sending the rising or falling voltage signal to the bus, the virtual time master Host 1 handles the signal on the voltage bus in the same way as Host2, Host3, and Host4 do. Therefore, no bias occurs at the virtual time master through any inadvance pause/resume operations. In addition, any host in Figure 5 is allowed to take the role of the virtual time master to request pause and resuming operations. Throughout the life of an experiment, many hosts take turns to serve as the virtual time master as they require an offline computation.

The design of the interrupts using rising and falling signals satisfies the case described in Figure 4. When multiple requests are created at the same time from distinct processes (across different devices), multiple signals can be sent out simultaneously. However, since all pins are connected to a common voltage bus, the rising signal interrupt is only triggered once; Similarly, the falling signal interrupt is only triggered when all output pins are changed from 


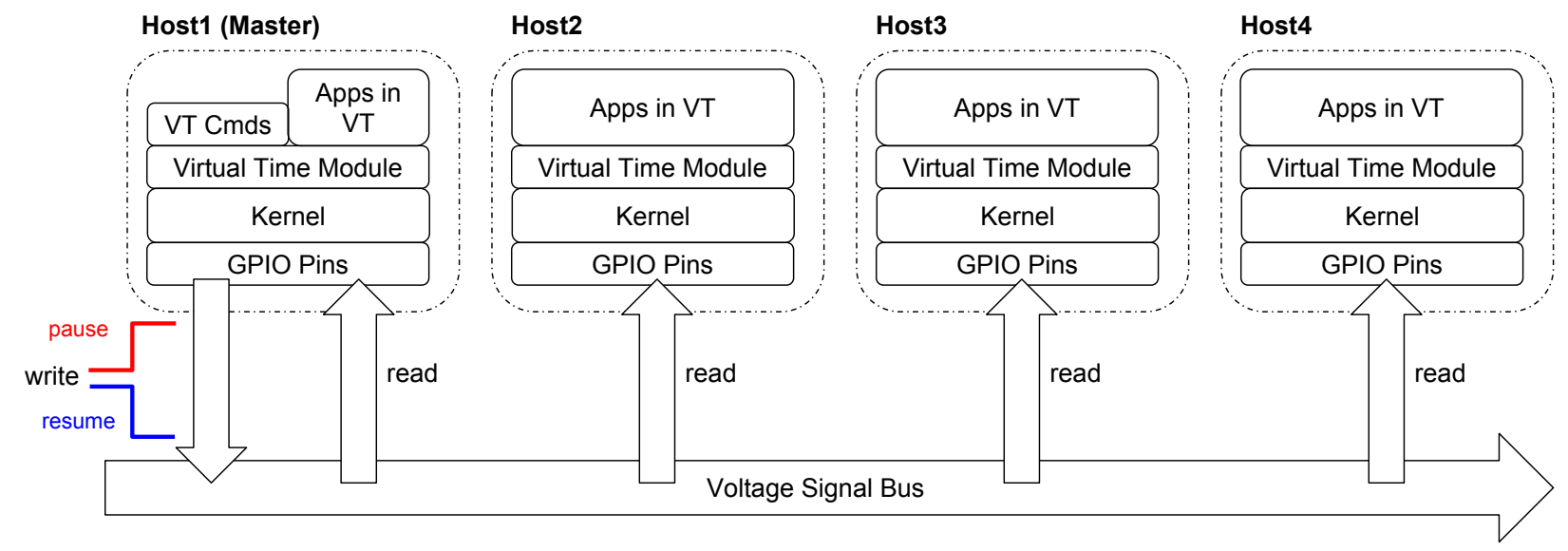

Figure 5: Distributed-Virtual-Time Enabled Emulation Testbed Built on Four Embedded Devices.

HIGH to LOW. Conveniently, the entire distributed testbed will initiate a pause at the moment of the first rising signal, equivalent to the pause criteria (1). Moreover, the testbed will not resume until the last signal changes to LOW triggering the falling interrupt matching the resume criteria (2). As a result, the signal bus frees the kernel module from having to deal with overlapping synchronization requests.

\subsection{Virtual Time Linux Kernel Module}

Figure 6 depicts the detailed implementation of the virtual time LKM, specifically for an embedded Linux system with GPIO support (i.e., the Banana PI single board computer). The virtual time LKM works in two phases, namely proactive mode and reactive mode. In the proactive mode, virtual time LKM outputs a signal to the underlying bus; in the reactive mode, a hardware signal is sent to the virtual time LKM.

Controls in proactive mode are initiated from the user-space commands performing the following operations: 1) register a process in virtual time, 2) query the virtual time information for a given process, 3) dilate the time of one or more processes, 4) pause all processes registered in virtual time, 5) resume all processes registered in virtual time. After a process is registered in virtual time, LKM makes the rest of the virtual time commands available to the process. Regarding the query command (the purple arrow in Figure 6), the virtual time system utilizes the sysfs subsystem to return the values of all virtual-time-related variables of that process. Regarding the dilate command, sysfs invokes dilate(pid, tdf), which enables virtual time on a given process with a user-specified time dilation factor $t d f$. Pause and resume commands work in the same way (the orange arrow in Figure 6). For a pausing request, the virtual time sysfs triggers a callback to output a HIGH voltage signal on Pin 1 via GPIO signal management API. For a resume command, the output voltage signal returns to a LOW one.

The proactive mode, however, only handles the part of work to pause or resume the processes in virtual time. The other half is accomplished in the reactive mode when the voltage signal sent by the proactive virtual time LKM is received on Pin 2 and Pin 3. The red arrow depicts how a rising signal triggers pause(). During the initialization, the virtual time LKM maps Pin 2's GPIO to a software interrupt number, irq. In addition, it registers the software interrupt handler pause() for irq. Only upon detecting a rising signal on Pin 2 will the GPIO signal management trigger the pre-mapped software interrupt irq, which is handled automatically by the virtual time LKM using the pause() handler. Algorithm 1 describes how the system pauses the processes attached to the virtual clock.

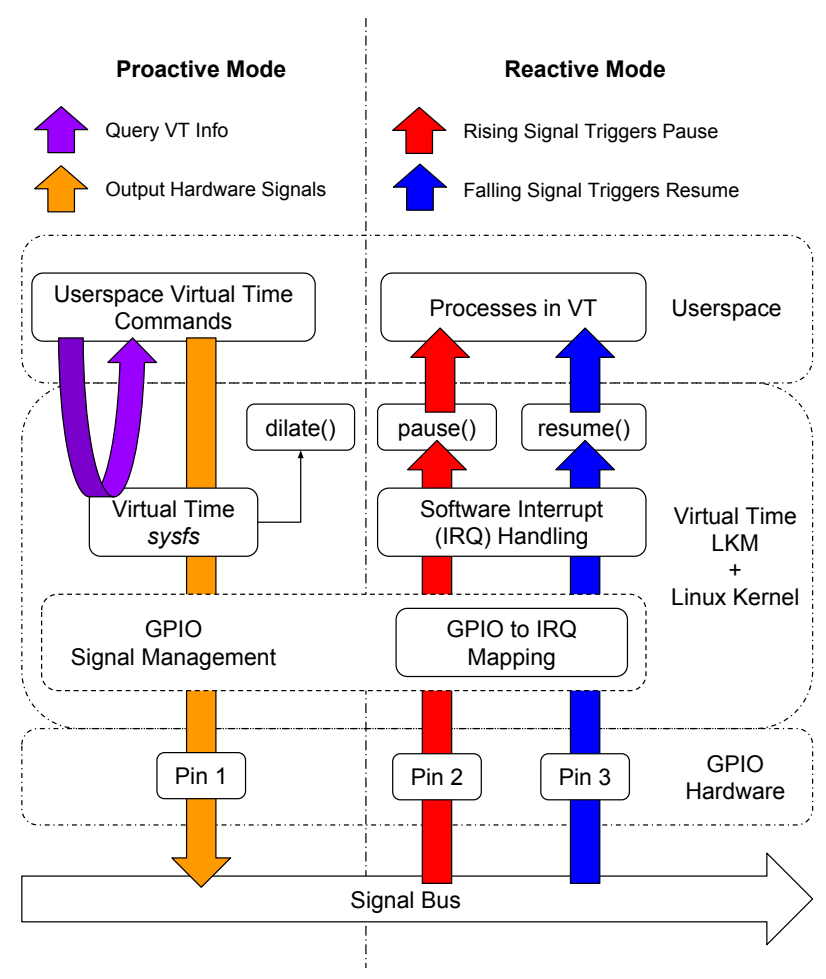

Figure 6: GPIO Signal and Software Interrupt Based Virtual Time Linux Kernel Module. 


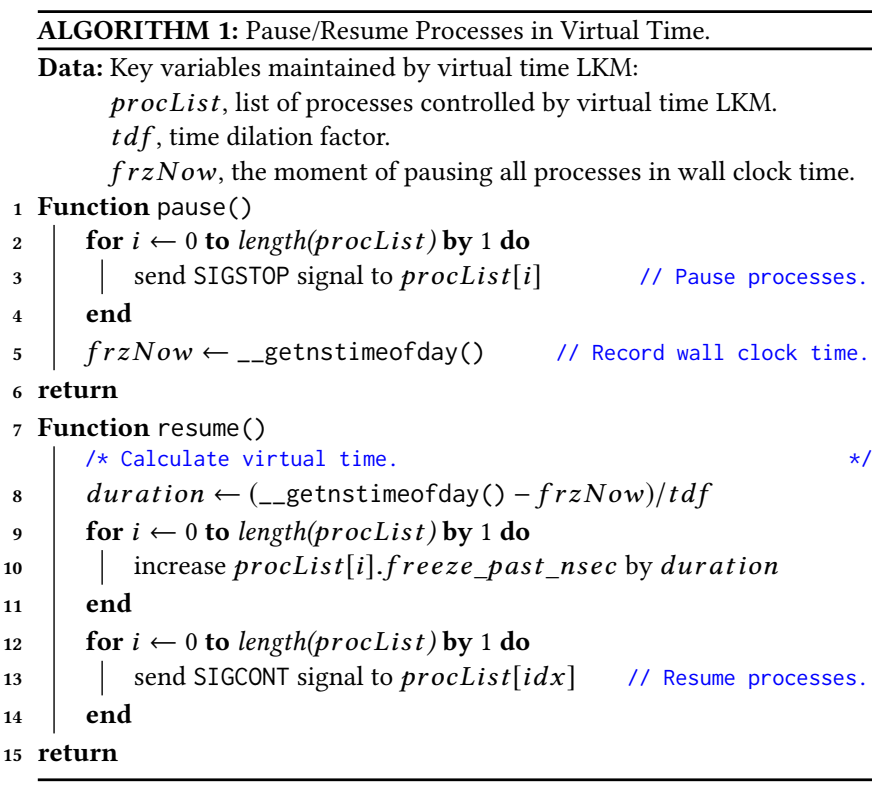

The system sends SIGSTOP signals to all virtual-time-enabled processes before querying the current wall clock time. In the case of the resuming operation (shown in blue), the differences are 1) the source is a falling voltage detected on Pin 3, and 2) the destination is another pre-registered software interrupt handler resume(), listed in Algorithm 1, for a separate software interrupt number $i r q^{\prime} \neq$ irq. Software interrupt handler resume() first calculates the duration since the pause moment in virtual time and then updates the variable freeze_past_nsec for each process. This way, all processes running on a single host are conceptually paused for the same duration of virtual time. Then the SIGCONT signal commands the kernel to wake up all processes registered in virtual clock. Even if the moments to wake up are different for processes in procList, the perceived pause duration is identical among the processes. Our signal-bus-based hardware design also simplifies Algorithm 1 as it is not necessary for the software module to cope with the tangled synchronization requests from multiple devices.

Our implementation works for various embedded Linux devices including the Banana Pi M1s, the Banana Pi R1 Routers, and the Raspberry Pi devices. Additionally, our solution is designed to be compatible with any hardware that runs Linux and has the GPIO programmability.

Barebone devices, such as 8- and 32-bit microcontrollers, are also compatible but some system-specific modification is required due to the lack of an operating system. Microcontrollers can enable emulation of full sensor and actuator components of cyber-physical systems. We leave this for future work.

\subsection{Virtual Time Retrieval}

In addition to the coordination of the virtual time LKM described in Section 3.2, to correctly retrieve virtual time we also modify the system calls that return time to a process. When a process requests time through the gettimeofday() system call, the kernel returns a virtual time if the requesting process is a virtual-time-enabled process. We port the virtual time kernel in [29] to the ARM Linux kernel 3.14. Our implementation behaves the same as the original kernel with necessary modifications regarding the ARM instruction set. The new virtual time kernel adds the file system entries to the /proc/\$PID directory to contain the virtual time metadata. When a process calls gettimeofday(), the function checks the virtual time metadata and returns the virtual time to a process. A major advantage of this approach is that the virtual time retrieval procedure is transparent to applications. The only requirement is that the time source must use the monotonic clock representation. The detailed kernel implementation is described in [29].

\section{EVALUATION}

In order to show the practicality of distributed virtual time, the overhead needs to be taken into account. Additionally, one needs to verify the correctness of the virtual time. The testbed used to evaluate the virtual time kernel module is made from 8 Banana PI devices. 4 devices are M1 single board computers and 4 devices are R1 embedded routers. Banana PI devices have the A20 ARM Cortex-A7 Dual-Core CPU with 1GB DDR3 Memory and a 1Gb Ethernet controller.

\subsection{Virtual time overhead}

The overhead can be divided into two components: the overhead added to the gettimeofday() system call and the overhead in pausing and resuming processes. To measure the overhead of the gettimeofday() system call, we employ the following two methods.

4.1.1 System Call gettimeofday(). The first way to measure overhead is the function tracer ftrace, which enables fine-grained measurements of kernel function latencies. ftrace, specifically the function graph tracer, works by probing a function on both its entry and exit using a dynamically allocated stack of return addresses, which it overwrites to calculate latencies [24]. We compile the kernel with the ftrace option to measure the overhead of virtual time related system calls. Table 1 illustrates the system call progression in and out of virtual time for the gettimeofday() system call. This is the overhead a process experiences when querying the clock. Virtual time clocks are transparent to the processes. A virtual time is returned in place of a real time. Virtual-time-based timekeeping in the gettimeofday () system call increases the kernel space overhead from an average of 8.625 microseconds to 13.333 microseconds. Seemingly virtual time adds substantial overhead in calculating the offset from the real clock.

The function tracer ftrace only monitors the kernel function sys_gettimeofday(). In order to measure the total time from the user space call, another method is employed. We create a $\mathrm{C}$ program that calls gettimeofday() 1,000,000 times and calculates the average duration of the call for a process registered to virtual time and a non-registered process. When not in virtual time, the average overhead of a regular process calling gettimeofday() is 11.833 microseconds per call, while in virtual time is 17.387 microseconds. For comparison, ext4_mark_inode_dirty () requires 58 microseconds, unlock_new_inode() 36 microseconds, and writing 2.7 MB to a file takes 975 microseconds with sys_write(). 
Virtual-time-enabled process

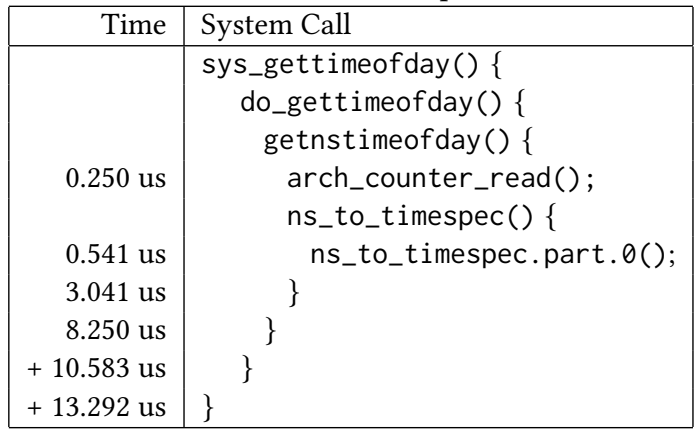

Regular non-virtual-time-enabled process

\begin{tabular}{|c|c|}
\hline Time & System Call \\
\hline & $\begin{array}{l}\text { sys_gettimeofday () }\{ \\
\text { do_gettimeofday () }\{ \\
\text { getnstimeofday () }\{\end{array}$ \\
\hline 0.250 us & arch_counter_read(); \\
\hline 2.792 us & \} \\
\hline 5.333 us & \} \\
\hline 8.000 us & \} \\
\hline
\end{tabular}

Table 1: The duration of the function is shown on the return from the call. The durations include the time of nested calls. (Top) Function trace is for gettimeofday () system call for virtual-time-enabled processes; The total time is $13.292 \mathrm{mi}$ croseconds. (Bottom) Function trace is for regular processes not registered to virtual time. The total time is 8.000 microseconds.

4.1.2 Linux Kernel Module Proactive Mode. As explained in the previous sections, and illustrated in Figure 6, there is a proactive mode and a reactive mode of the Linux Kernel Module (LKM). In the proactive mode, a process writes to the virtual file system to trigger a callback that changes the output on GPIO Pin 1. We employ ftrace to measure the latency in the LKM including writing to the virtual file system. The main function in the kernel in the proactive mode is sys_write(). This function takes on average 262.292 microseconds. The main functions sys_write() calls are: sysfs_write_file() (204.5 microseconds), which calls mode_store() (105.833 microseconds) and in turn either calls gpio_direction_output () (47.250 microseconds) or gpio_direction_in () (38.833 microseconds) for pause and resume functions respectively. The top level function writes a value to the virtual file system /sys/VT/mode, which determines if pause or resume should be called inside the LKM. Next mode_store () changes the state of the GPIO Pin 1. A full trace of a proactive pause routine can be found in the online repository due to the length.

4.1.3 Linux Kernel Module Reactive Mode. Tracing the reactive mode of the LKM is more difficult as the function tracer is unable to probe the entrance and exit of the interrupt functions. In order to measure the overhead of the reactive component of the LKM, we timestamp the entrance into and exiting the pause and resume

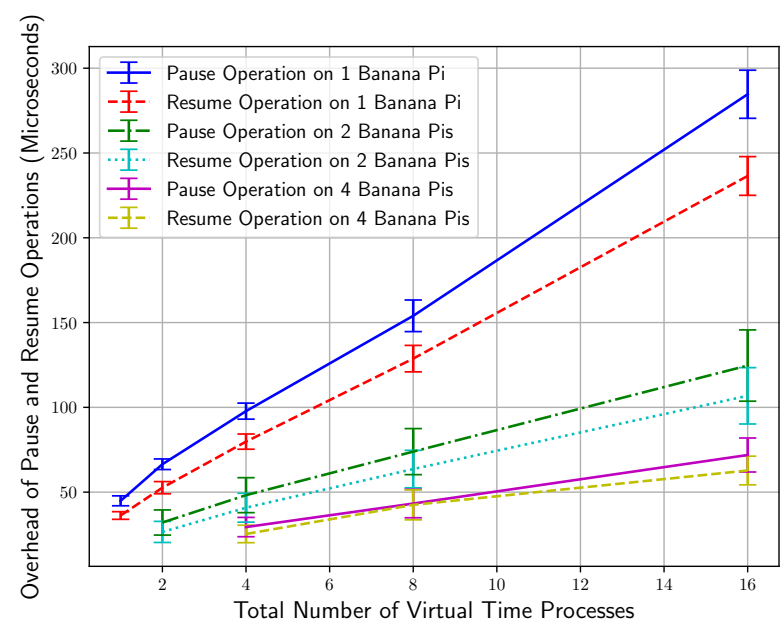

Figure 7: The overhead of pausing and resuming scales linearly with the number of processes subscribed to virtual time. Using more boards allows the parallelization of virtual-time-enabled processes, which enables a linear speedup as expected.

functions. For each process registered in virtual time, the LKM sends a SIGSTOP or SIGCONT signal. Additionally, it writes some timekeeping data to the processes /proc/\$PID/ directory. Figure 7 illustrates the overhead of pausing and resuming virtual time processes in the reactive mode of the LKM. The overhead of a single machine, 2 machines, and 4 machines are plotted for up to 16 virtual time processes with their $95 \%$ confidence intervals. The overhead scales linearly with the number of virtual-time-enabled processes for all scenarios. On a single machine, 16 virtual-time-enabled processes take 282 microseconds to pause and 234 microseconds to resume. Using more boards allows us to parallelize the pausing and resume, which downscales the overhaed as shown in Figure 7.

\subsection{Virtual Time Correctness}

In addition to determining the latency imposed by virtual time, it is also critical that the virtual time does not introduce unexpected errors in applications. To evaluate the correctness of the distributed virtual time system, it is important that the systems' clocks do not skew over time. Additionally, we evaluate the bandwidth between two Banana Pi hosts in and out of virtual time to ensure that errors are not introduced.

4.2.1 Clock Skewness. The clock skewness is the difference in the value of time reported by two clocks at a given instance in time. Two factors can contribute to the clock skewness. Firstly as in all commodity systems, the onboard clock is not perfect, the clocks on the Banana PIs will naturally drift with time. The Network Time Protocol (NTP) can be used to correct drift errors. The second source of clock skewness is introduced by the virtual time system. Since it is infeasible to query two clocks simultaneously with high fidelity to determine the clock skewness, we employ an indirect method. The virtual time is given by the wall clock time minus paused 


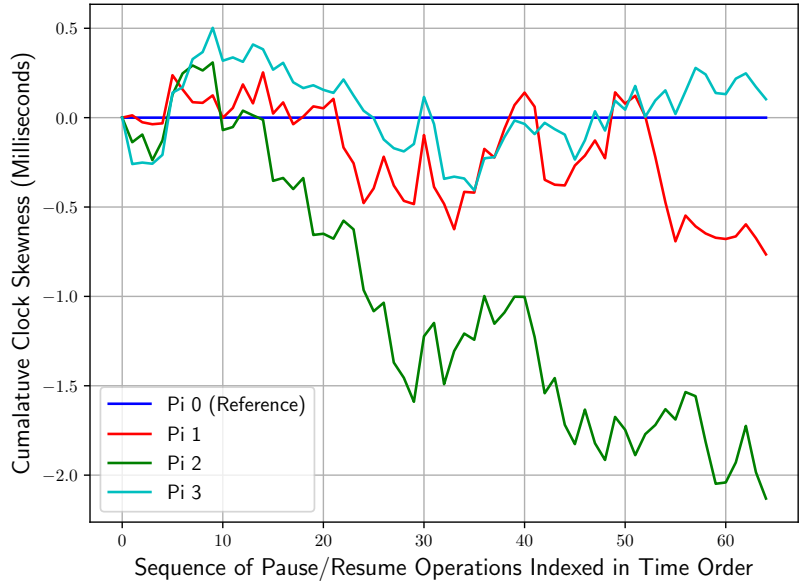

Figure 8: The processes are periodically paused and resumed using the virtual time system. We measure the skewness in milliseconds on the $y$-axis and plot the index of the pause/resume operations on the $x$-axis. The processes are paused and resumed 64 times. The skewness of the clocks over time remains within acceptable limits in comparison with typical clock skewness. The skewness is measured by querying the cumulative paused time of each virtual-timeenabled process.

time, and one can query the paused time while the virtual time processes are running. Additionally, paused time advances only when processess are paused and resumed. To evaluate the clock skewness, we apply the pause and resume operations on 4 virtualtime-enabled processes across 4 Banana Pi devices. Each process within a device shares the same virtual time offset. Each device takes its turn as the master node shown in Figure 5 in a round robin order. After each resume operation, the cumulative paused time is recorded by querying the virtual time interface. In Figure 8 , we plot the skewness of the virtual time clocks with respect to an arbitrary virtual time $\mathrm{Pi}$ as the reference. The results show that the clocks do skew over time but that they remain within a close range after 64 pause and resume operations. In [20], the authors examine clock drift over Internet-of-things devices including Raspberry Pi devices, which are similar to the Banana Pi devices used in our work. The authors conclude that with their implementation they can maintain a clock accuracy of within 15 milliseconds. Using NTP, the clock skewness we observe in virtual time remains tolerable. Our future work involves consideration of out-of-band clock synchronization algorithms for the virtual clocks similar to NTP for the processors in the wall clock.

4.2.2 Network Performance in Virtual Time. In order to verify the correctness of our virtual time system, we measure the performance of a common networking application in and out of virtual time. We connect two Banana Pi hosts together via Ethernet and run iperf3 in TCP mode. In the first case, we run iperf for $30 \mathrm{sec}-$ onds with processes not in virtual time. Although the network

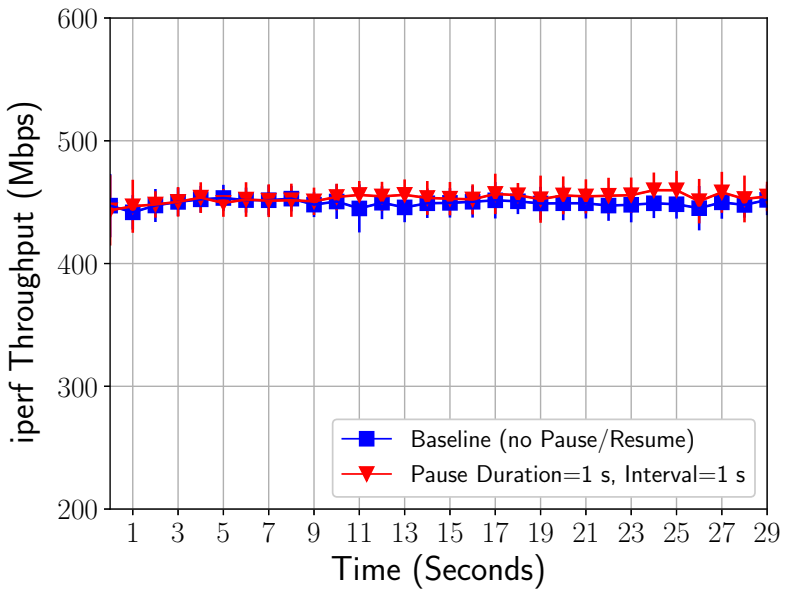

Figure 9: We use iPerf to measure the bandwidth between two hosts. This graph shows that the performance of the networking application iPerf is correct when a process is in virtual time.

interface is rated for $1 \mathrm{Gbps}$, we see that the practical limit is closer to $450 \mathrm{Mbps}$. In the second case, we add the iperf processes on both hosts to virtual time and periodically pause and resume from the iperf client. The virtual time system is completely transparent to the iperf applications. The client host is paused every 1 second for a duration of 1 second. The iperf test is also run for 30 virtual time seconds, which takes 60 wall clock seconds. The bandwidth we observe in Figure 9 is similar to the base case where processes are not using virtual time.

\section{CYBER-PHYSICAL SYSTEM USE CASE}

One of the largest cyber-physical systems is the electric power grid, which is compromised of many physical components including generators, transformers, loads, capacitors, etc. Due to the emergence of distributed and renewable generation such as solar power and wind generation, maintaining the stability of the electric power grid is becoming increasingly important and expensive. When electric energy generation is dynamic due to fluctuations in the physical environment, electric grid operators must implement appropriate strategies including demand response [25], virtual power plants [23], energy storage systems [3], etc. These techniques require greater observability and controllability, which in turn, requires more extensive communication infrastructure within the power grid.

\subsection{Voltage Stability Application}

We demonstrate the usability of our virtual-time-enabled testbed by evaluating the cyber-security of a voltage stabilization application. In order to maintain grid stability, an actuator compensates for the changes in the dynamic generation in real-time. It consists of an energy storage device and a control system, and works as follows. A sensor collects voltage and frequency data from the distributed energy resource in the electric power grid. It transmits the data to the actuator's control system that determines the required settings 


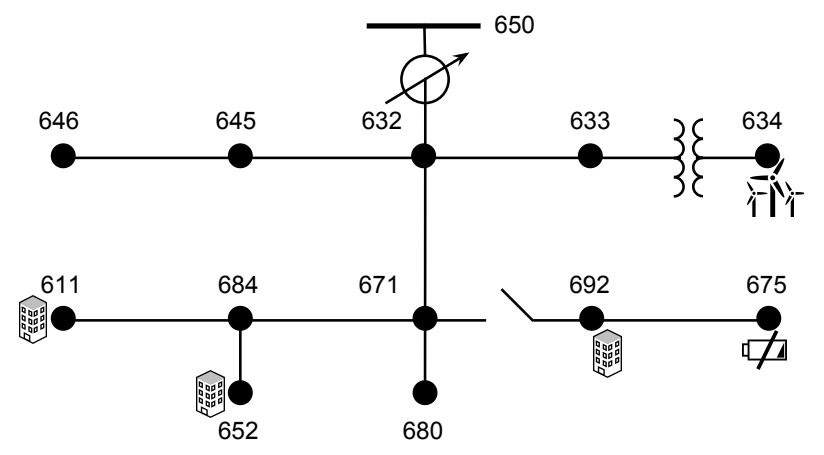

Figure 10: 13-Bus Distribution System

for the energy storage device. If there is an excess of energy being produced, the control system will reduce the discharge rate of the energy storage device. If the excess is large, the energy storage device will switch from discharge to charge mode to consume energy and replenish its storage. On the other hand, if the energy produced from the generator is low, the energy storage device will supplement the grid with power.

In this use case, we consider the voltage stabilization application run in the IEEE 13-bus power system [15] illustrated in Figure 10. The power system is composed of busses, which are nodes in the power system topology, such as substations which distribute power to loads to residential communities. Busses also connect generators to the power grid, such as solar, wind, and hydro resources. The IEEE 13-Bus test case is used with added dynamic loads at Bus 611, Bus 652, and Bus 692. A dynamic generator is added at Bus 634 and an energy storage device is added at Bus 675 . The point of common coupling to the transmission network is at Bus 650 . The base system voltage is $2.4 \mathrm{kV}$ provided from this bus.

The cyber components are run on our distributed virtual time testbed while the physics of the power system is simulated. We adopt a linear communication network topology to connect the hosts in the control application. Figure 11 illustrates the linear communication network we created using the Banana Pi hosts and routers to emulate the cyber-components of the system. The energy storage device (Host 1 ) is connected to switch s1 while the sensor measuring generation output (Host 2) is connected to switch s4. All hosts are additionally connected to a power grid simulator, OpenDSS $[4,21]$, through a separate network to simulate the physical state of the system. The power simulator is run on a Windows 10 virtual machine with $8 \mathrm{~GB}$ of memory and four 4 $\mathrm{GHz}$ cores. The sensor retrieves voltage, frequency, and current data from the power simulator, and the actuator changes the state of the system by modifying the state of the energy storage device. The simulator advances when either the sensor requests data or when the energy storage device changes the output state. The simulator updates and advances its state up to the virtual clock time at a millisecond time step. Emulation experiment takes up to 10 times as long as the wall clock advances, due to the computational complexity of both calculating the state changes and sampling the circuit properties of the electric power system.

When the sensor determines it is time to retrieve a value from the power simulator, it sends its request to a management process that makes the call to the virtual time module. The management process interfaces with the power simulation server to relay the request. When the request is fulfilled, the management process places the sensor data in the sensor process's memory. The final step is to interact with the virtual time system to resume the processes as in Figure 6.

The blue line in Figure 12 shows the operation of the power grid under the normal behavior. The upper right plot shows the variability in the generation of the wind turbine over time in apparent power. The lower right plot illustrates the functionality of the voltage stability application over 90 seconds. The energy storage device charges for a short period between 18 and 21 seconds while discharging at a variable rate for the remainder of the experiment. Since there still remains randomness in the quantity of power consumed by loads, there are some voltage fluctuations as can be seen in the left two figures, which illustrates the voltage at Bus 680 and Bus 646 in the power system. However, the voltage does not deviate significantly from the baseline of 2400 Volts.

\subsection{Cyber-Attacks on Power Grid}

We demonstrate how a cyber-attack can affect the voltage stability of the power system, and impact the security of the power application. We make the assumption that a malicious actor has access to a compromised device on the network. Using dsniff [26], the attacker floods the ARP tables of the devices on the network in order to 1) convince the sensor that the attacker is the energy storage device's controller and 2) convince the energy storage device's controller that it is the sensor. Equivalently, the attacker is able to eavesdrop on all communication between the sensor and the controller. Furthermore, by removing the compromised host's network port from promiscuous mode, the attacker can create a denial-of-service (DoS) attack preventing network traffic from routing between the honest hosts. In Figure 12, the red curves illustrate the case when the attacker performs a DoS attack at time $22.5 \mathrm{sec}-$ onds. After this point, the communication channel between Host 1 and Host 2 is severed. Because the energy storage device is unable to receive new control signals, it maintains its state of discharging. The effects of the DoS attack can be seen in the figures on the left. At Bus 680, the voltage deviates 3.33 percent from nominal to 2320 volts. If the grid experiences instability for a prolonged period of time, the frequency of the power system will deviate, which causes larger generators to protectively shut down, further contributing to voltage instability and power outages. Because of the magnitude of the power deficit, the voltage drop at the busses will follow the generator's output.

After an attacker has access to the network, and is able to eavesdrop on real-time traffic, there is the potential for spoofing attacks, replay attacks, false data injection attacks, and more. Spoofing attacks can impersonate traffic to send data, configuration, or even control messages to cyber-physical devices in the grid, potentially opening relays or putting the grid in an unstable state. Replay attacks enable the attacker to resend network packets that have the potential to change the state of the system. Even encrypted packets have a potential to become susceptible to this family of attacks. Furthermore, by modifying packets in transit, applications 


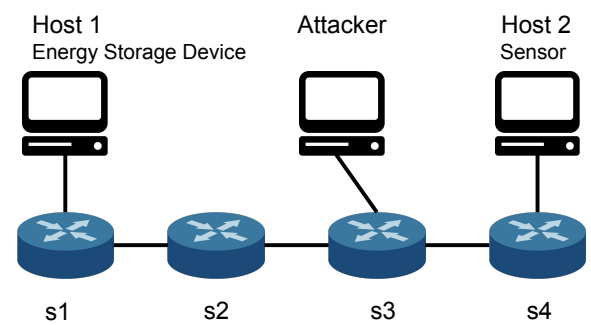

Figure 11: The communication network used in the power grid application.

can become victims to false data injections that force control applications to make improper decisions. There exist preventative and reactionary measures to prevent such attacks. For example, having a static routing policy at the switch level can prevent ARP spoofing and poisoning attacks. Encryption under proper configuration can mitigate eavesdropping, and spoofing attacks. The more cyber components that are adopted, the more cyber security plays a role in creating resilient and robust systems.

In summary, we demonstrate the role of distributed virtual time in a physical testbed equipped with simulation modeling using a voltage stability application. The distributed virtual time is critical because simulating the actuator's control system is computationally intensive and cannot be performed in real time. With distributed virtual time, it runs in synchrony with the physical networking system, which is a must-have for reproducing various attacks on power grid launched from its underlying networking layer, and for evaluating possible countermeasures.

\section{RELATED WORK}

Research work on virtual time in network emulation can be generally classified into two categories based on the application objectives. The first objective is to improve the scalability and fidelity of network emulation testbeds. They typically adopt the virtual time technique to uniformly scale the emulation entity's perception of time by a specified factor. It was first introduced as time dilation in [9], and has been adopted to various types of virtualization techniques (e.g., virtual machines, virtual nodes, Linux containers) and integrated with a handful of network emulators $[5,6,8,18,30]$. For example, VT-Mininet [30] used time dilation to virtually scale up the system resources on a single commodity machine to support high-fidelity analysis of large-scale software-defined networks. TimeKeeper [17] studied how time dilation enables "moderate hardware" to emulate a smart grid control network that requires "powerful hardware." In addition, [7] explored means to minimize the running time of dilated network emulation experiments based on the historical average resource requirements of virtual nodes.

The second objective is on the temporal integration of network emulation and simulation. Several hybrid testing systems that integrate network emulation and simulation have adopted this approach $[12,14,18,28]$. For example, [11] combined a power distribution system simulator with an SDN emulator to support evaluation of communication network applications and their impacts on corresponding power systems. The synchronization of the two systems is achieved via freezing (and then resuming) Linux containers including their own virtual clocks. By embedding Linux processes in virtual time, TimeKeeper [18] successfully integrated two network simulators (ns-3 and S3F) to a network emulator with negligible modification. SliceTime [28] used a common barrier to block both simulation processes and virtual machines in emulation until both systems complete a rational time slice.

Distributed virtual time relies on software triggering hardware interrupts to coordinate and synchronize between cyber and physical simulation systems. Interrupts are also used in simulation systems including parallel systems to preempt events [22]. However, the previous uses of interrupts are used to synchronize more efficiently in optimistic simulation while our use is to uniformly halt the cyber system.

Distributed virtual time facilitates the integration of simulation to emulated or physical hardware based testbeds that are temporally transparent to the emulated processes. This paper presents the first working virtual time system that is synchronized across multiple physical devices.

\section{CONCLUSION}

Planning and evaluation of cyber-physical systems requires highfidelity simulation and emulation testing platforms. Virtual time is a powerful technique for integrating simulation and emulation models. In this paper, we propose a distributed version of virtual time across multiple embedded Linux devices, which are essential components of a cyber-physical system testbed. Through the use of hardware interrupts across a common voltage bus, multiple embedded Linux devices can be synchronized in virtual time. Each device runs a Linux kernel module that supports distributed virtual time for processes across devices. We show how we minimize system overhead in pausing and resuming virtual time processes. Additionally, we show that the performance of the iperf networking application remains the same when using virtual time. Through a use case, we demonstrate the usability of a distributed virtualtime-enabled testbed for evaluating the cyber-security of a voltage control application in the electric power grid. In our future work, we will further evaluate cyber-physical system operations using the testbed supporting distributed virtual time. Specifically, we would like to evaluate the impact of disruptions in the communication network for frequency adjustment control applications in the electric power grid as this is a cyber-physical system application that utilizes real-time sensing and actuation.

\section{ACKNOWLEDGMENT}

This work is partly sponsored by the Air Force Office of Scientific Research (AFOSR) under Grant YIP FA9550-17-1-0240, the National Science Foundation (NSF) under Grant CNS-1730488, and the Maryland Procurement Office under Contract No. H98230-18-D0007. Any opinions, findings and conclusions or recommendations expressed in this material are those of the author(s) and do not necessarily reflect the views of AFOSR, NSF, and the Maryland Procurement Office.

\section{REFERENCES}

[1] Allwinner Technology Co., Ltd. 2013. CoreLink GIC-400 Generic Interrupt Controller: Technical Reference Manual (1.0 ed.). Allwinner Technology Co., 

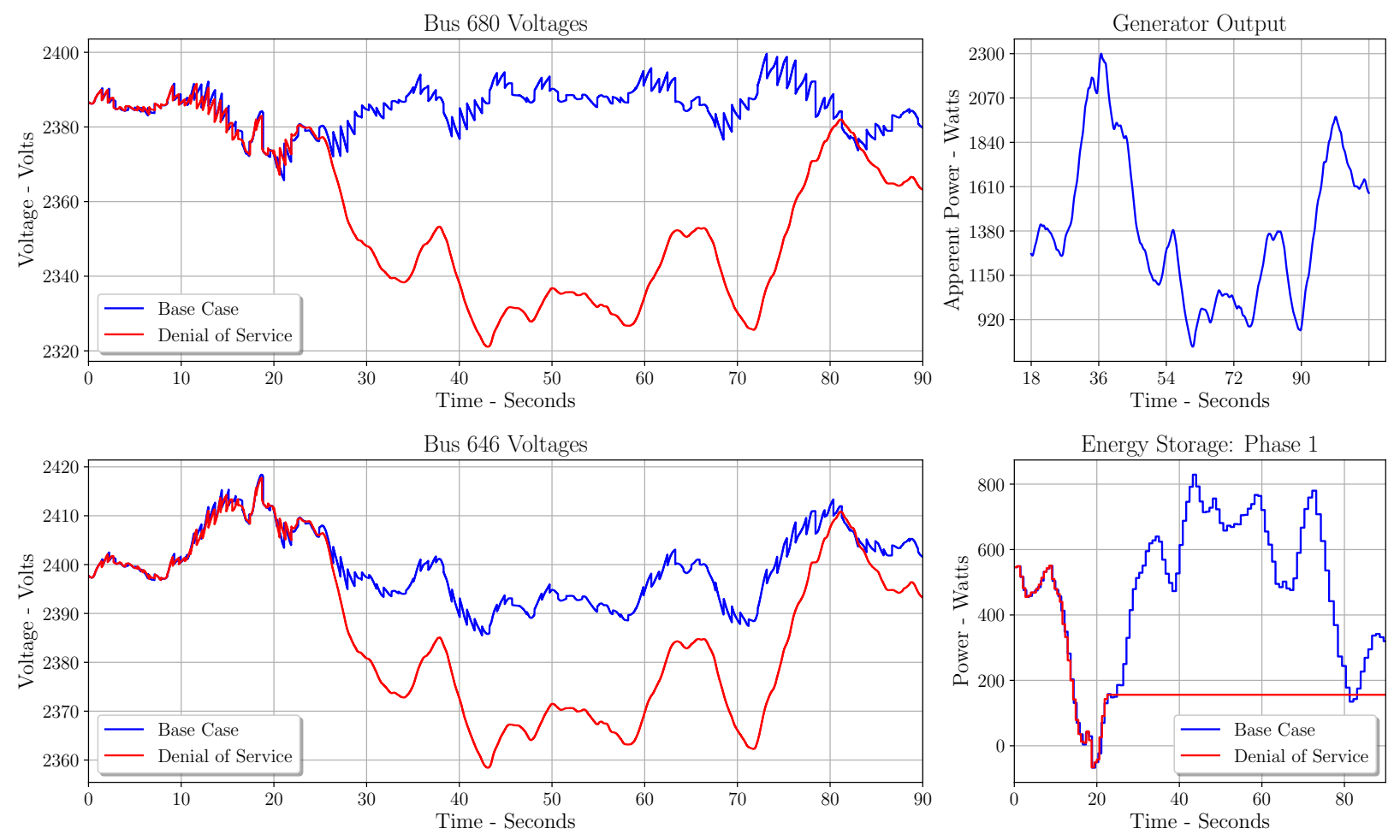

Figure 12: Left: voltage of the power system at bus 680 (Up) and bus 646 (Down). Top Right: The apparent power in Watts generated by the wind turbine. Bottom Right: The energy storage device's supplied power and load.

Ltd. http://infocenter.arm.com/help/topic/com.arm.doc.ddi0471a/DDI0471A_ gic 400 r0p0 trm.pdf.

[2] ARM 2011. A20 User Manual (r0p0 ed.). ARM. http://dl.linux-sunxi.org/A20/ A20UserManual2013-03-22.pdf.

[3] J. M. Carrasco, L. G. Franquelo, J. T. Bialasiewicz, E. Galvan, R. C. PortilloGuisado, M. A. M. Prats, J. I. Leon, and N. Moreno-Alfonso. 2006. Power-Electronic Systems for the Grid Integration of Renewable Energy Sources: A Survey IEEE Transactions on Industrial Electronics 53, 4 (June 2006), 1002-1016. https: //doi.org/10.1109/TIE.2006.878356

[4] Roger C Dugan. 2013. Reference Guide, The Open Distribution System Simulator (2013).

[5] M.A. Erazo, Yue Li, and J. Liu. 2009. SVEET! a scalable virtualized evaluation environment for TCP. In Proceedings of the 2009 Testbeds and Research Infrastructures for the Development of Networks Communities and Workshops. IEEE Computer Society, Washington, DC, USA, 1-10.

[6] A. Grau, K. Herrmann, and K. Rothermel. 2011. NETbalance: Reducing the Runtime of Network Emulation Using Live Migration. In Proceedings of the 20th International Conference on Computer Communications and Networks. IEEE Computer Society, Washington, DC, USA, 1-6.

[7] Andreas Grau, Klaus Herrmann, and Kurt Rothermel. 2012. Scalable Network Emulation - The NET Approach. Fournal of Communications 7, 1 (January 2012), $3-16$.

[8] Andreas Grau, Steffen Maier, Klaus Herrmann, and Kurt Rothermel. 2008. Time Jails: A Hybrid Approach to Scalable Network Emulation. In Proceedings of the 22nd Workshop on Principles of Advanced and Distributed Simulation. IEEE Computer Society, Washington, DC, USA, 7-14.

[9] Diwaker Gupta, Kenneth Yocum, Marvin McNett, Alex C. Snoeren, Amin Vahdat, and Geoffrey M. Voelker. 2005. To Infinity and Beyond: Time Warped Network Emulation. In Proceedings of the 20th ACM Symposium on Operating Systems Principles. ACM, New York, NY, USA, 1-2.

[10] Christopher Hannon, Jiaqi Yan, and Dong Jin. 2016. DSSnet: A Smart Grid Modeling Platform Combining Electrical Power Distribution System Simulation and Software Defined Networking Emulation. In Proceedings of the 2016 ACM SIGSIM Conference on Principles of Advanced Discrete Simulation (SIGSIM-PADS '16). ACM, New York, NY, USA, 131-142. https://doi.org/10.1145/2901378.2901383
[11] Christopher Hannon, Jiaqi Yan, and Dong Jin. 2016. DSSnet: A Smart Grid Modeling Platform Combining Electrical Power Distribution System Simulation and Software Defined Networking Emulation. In Proceedings of the 2016 ACM SIGSIM Conference on Principles of Advanced Discrete Simulation (SIGSIM-PADS '16). ACM, New York, NY, USA, 131-142. https://doi.org/10.1145/2901378.2901383

[12] Christopher Hannon, Jiaqi Yan, Dong Jin, Chen Chen, and Jianhui Wang. 2018. Combining Simulation and Emulation Systems for Smart Grid Planning and Evaluation. ACM Trans. Model. Comput. Simul. 28, 4, Article 27 (Aug. 2018), 23 pages. https://doi.org/10.1145/3186318

[13] Dong Jin, Yuhao Zheng, Huaiyu Zhu, David M Nicol, and Lenhard Winterrowd. 2012. Virtual time integration of emulation and parallel simulation. In Proceedings of the 2012 ACM/IEEE/SCS 26th Workshop on Principles of Advanced and Distributed Simulation. IEEE Computer Society, 201-210.

[14] Dong Jin, Yuhao Zheng, Huaiyu Zhu, David M. Nicol, and Lenhard Winterrowd. 2012. Virtual Time Integration of Emulation and Parallel Simulation. In Proceedings of the 2012 ACM/IEEE/SCS 26th Workshop on Principles of Advanced and Distributed Simulation. IEEE Computer Society, Washington, DC, USA, 201-210.

[15] W. H. Kersting. 2001. Radial distribution test feeders. In 2001 IEEE Power Engineering Society Winter Meeting. Conference Proceedings (Cat. No.01CH37194), Vol. 2. 908-912 vol.2. https://doi.org/10.1109/PESW.2001.916993

[16] Jereme Lamps, Vladimir Adam, David M. Nicol, and Matthew Caesar. 2015. Conjoining Emulation and Network Simulators on Linux Multiprocessors. In Proceedings of the 3rd ACM SIGSIM Conference on Principles of Advanced Discrete Simulation (SIGSIM PADS '15). ACM, New York, NY, USA, 113-124. https: //doi.org/10.1145/2769458.2769481

[17] Jereme Lamps, Vignesh Babu, David M. Nicol, Vladimir Adam, and Rakesh Kumar. 2018. Temporal Integration of Emulation and Network Simulators on Linux Multiprocessors. ACM Trans. Model. Comput. Simul. 28, 1, Article 1 (Jan. 2018), 25 pages. https://doi.org/10.1145/3154386

[18] Jereme Lamps, David M. Nicol, and Matthew Caesar. 2014. TimeKeeper: A Lightweight Virtual Time System for Linux. In Proceedings of the 2Nd ACM SIGSIM Conference on Principles of Advanced Discrete Simulation (SIGSIM PADS '14). ACM, New York, NY, USA, 179-186. https://doi.org/10.1145/2601381.2601395

[19] Bob Lantz, Brandon Heller, and Nick McKeown. 2010. A network in a laptop: rapid prototyping for software-defined networks. ACM Press, 1-6. https://doi. org/10.1145/1868447.1868466 01341 . 
[20] Sathiya Kumaran Mani, Ramakrishnan Durairajan, Paul Barford, and Joel Sommers. 2018. An Architecture for IoT Clock Synchronization. In Proceedings of the 8th International Conference on the Internet of Things (IOT '18). ACM, New York, NY, USA, Article 17, 8 pages. https://doi.org/10.1145/3277593.3277606

[21] Davis Montenegro, Roger Dugan, Robert Henry, Tom McDermott, and wsunderm1. 2016. OpenDSS Program, SOURCEFORGE.NET. http://sourceforge.net/ projects/electricdss. (2016). [Last accessed January 2016].

[22] Alessandro Pellegrini and Francesco Quaglia. 2017. A Fine-Grain Time-Sharing Time Warp System. ACM Trans. Model. Comput. Simul. 27, 2, Article 10 (May 2017), 25 pages. https://doi.org/10.1145/3013528

[23] D. Pudjianto, C. Ramsay, and G. Strbac. 2007. Virtual power plant and system integration of distributed energy resources. IET Renewable Power Generation 1, 1 (March 2007), 10-16. https://doi.org/10.1049/iet-rpg:20060023

[24] Steven Rostedt. 2017. ftrace - Function Tracer (4.13 ed.). Red Hat Inc. https://www.kernel.org/doc/Documentation/trace/ftrace.txt.

[25] Pierluigi Siano. 2014. Demand response and smart gridsâĂŤA survey. Renewable and Sustainable Energy Reviews 30 (2014), 461 - 478. https://doi.org/10.1016/j. rser.2013.10.022

[26] Dug Song. 2001. dsniff. (Dec. 2001). Retrieved Janunary 21, 2019 from https: //www.monkey.org/ dugsong/dsniff/

[27] The Friends-of-Fritzing foundation. 2018. Fritzing. (Feb. 2018). Retrieved Janunary 10, 2019 from http://fritzing.org

[28] Elias Weingärtner, Florian Schmidt, Hendrik Vom Lehn, Tobias Heer, and Klaus Wehrle. 2011. SliceTime: A Platform for Scalable and Accurate Network Emulation. In Proceedings of the 8th USENIX Conference on Networked Systems Design and Implementation. USENIX Association, Berkeley, CA, USA, 253-266.

[29] Jiaqi Yan and Dong Jin. 2015. A Virtual Time System for Linux-container-based Emulation of Software-defined Networks. In Proceedings of the $3 \mathrm{rd}$ ACM SIGSIM Conference on Principles of Advanced Discrete Simulation (SIGSIM PADS '15). ACM, New York, NY, USA, 235-246. https://doi.org/10.1145/2769458.2769480

[30] Jiaqi Yan and Dong Jin. 2015. VT-Mininet: Virtual-time-enabled Mininet for Scalable and Accurate Software-Define Network Emulation. In Proceedings of the 1st ACM SIGCOMM Symposium on Software Defined Networking Research (SOSR '15). ACM, New York, NY, USA, Article 27, 7 pages. https://doi.org/10 $1145 / 2774993.2775012$

\section{APPENDIX}

Figure 13 illustrates how the GPIO pins are connected to form the signal bus. The number of GPIO pins required for distributed virtual time is 3 regardless of the number of boards in virtual time. The Raspberry Pi devices have a similar pinout to Banana Pi devices.

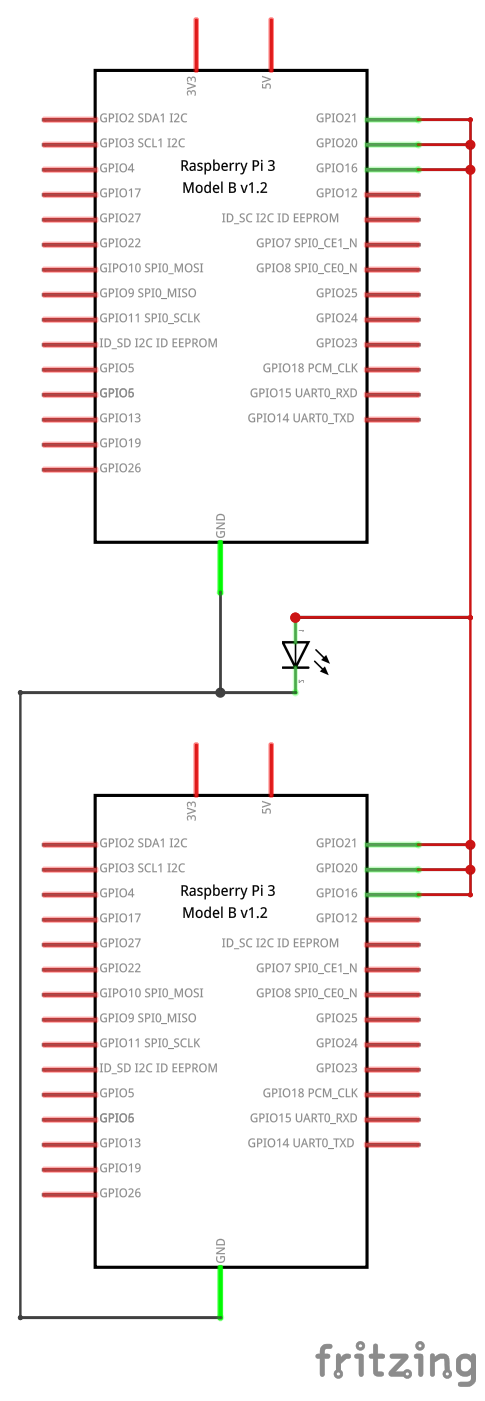

Figure 13: Three GPIO pins are used on each machine in the distributed virtual time testbed. In the schematic shown are two Raspbery Pi 3 Model Bs. The figure was drawn using the fritzing tool [27]. 